\title{
Clinical effect of continuous corrective force delivery in the non-operative treatment of idiopathic scoliosis: a prospective cohort study of the triac-brace
}

\author{
Gerben J. Bulthuis · Albert G. Veldhuizen • \\ Gert Nijenbanning
}

Received: 14 November 2006/Revised: 30 July 2007 / Accepted: 16 September 2007/Published online: 10 October 2007

(C) Springer-Verlag 2007

\begin{abstract}
A prospective cohort study of skeletally immature idiopathic scoliotic patients treated with the TriaC brace. To determine if the TriaC brace is effective in preventing curve progression in immature adolescent idiopathic scoliotic patients with a very high risk of curve progression based on reported natural history data. The aim of the newly introduced TriaC brace is to reverse the pathologic transverse force pattern by externally applied and continuously present orthotic forces. In the frontal plane the force system used in the TriaC brace is similar to the force system of the conventional braces. However, in the sagittal plane the force system acts only on the thoracic region. In addition, the brace allows upper trunk flexibility without affecting the corrective forces during body motion. In a preliminary study it is demonstrated that the brace prevents further progression of both the Cobb angle and axial rotation in idiopathic scoliosis. Skeletally immature patients with idiopathic scoliosis with curves between 20 and $40^{\circ}$ were studied prospectively. Skeletally immature was defined as a Risser sign 0 or 1 for both boys and girls, or pre-menarche or less than 1-year post-menarche for girls. Curves of less than $30^{\circ}$ had to have documented progression before entry. The mean age of the patients at the start of treatment was $11.3 \pm 3.1$ years. All measurements were collected by a single observer, and all patients
\end{abstract}

G. J. Bulthuis $(\varangle)$ · A. G. Veldhuizen

Department of Orthopaedics,

University Medical Center of Groningen,

Po.Box: 30.001, 9700 RB Groningen,

The Netherlands

e-mail: g.j.bulthuis@orth.umcg.nl

G. Nijenbanning

Director of Baat Engineering of Hengelo,

Hengelo, The Netherlands were followed up to skeletal maturity. Treatment was complete for all participants when they had reached Risser sign 4 and did not show any further growth at length measurements. This was at a mean age of $15.6 \pm 1.1$ years, with a mean follow-up of 1.6 years post bracing. In our study a successful outcome was obtained in $76 \%$ of patients treated with the TriaC brace. Comparing our data to literature data on natural history of a similar cohort shows that the TriaC brace significantly alters the predicted natural history. The current study demonstrates that treatment with the TriaC brace reduces the scoliosis, and that the achieved correction is maintained in some degree after skeletal maturity is reached and bracing is discontinued. It also prevents further progression of the Cobb angle in idiopathic scoliosis. The new brace does not differ from the conventional braces as far as maintaining the deformity is concerned.

Keywords Scoliosis - Biomechanics - Natural history · Bracing

\section{Introduction}

The purpose of this paper is to present the results of a prospective study of a new orthotic device for the non-operative treatment of idiopathic scoliosis. Data are provided in a format to facilitate comparison with natural history data.

The main concern in patients with idiopathic scoliosis relates to curve progression and the resulting cosmetic deformity. The risk of curve progression is correlated primarily to periods of rapid skeletal growth $[3,5,6,11,14$, $41,42]$. Factors related to growth potential, such as patient age at the time of diagnosis, status of menarche, and Risser 
sign, have been shown to be important predictors of the progression of scoliosis [3, 15, 29, 39]. In addition to future skeletal growth, curve magnitude and curve shape are further predictors of progression of idiopathic scoliosis. Large initial curves, thoracic curves, and double major curves are more likely to progress $[3,29,39]$.

Currently bracing is the accepted nonoperative treatment to prevent curve progression in mild to moderate scoliosis during the growth period. A prospective multicenter study, performed by the Scoliosis Research Society, reported that brace treatment has a significant effect on curve progression of idiopathic scoliosis [36]. However, others doubt the effectiveness of braces [12, 16, 19, 32]. Just as for the natural history of untreated curves, Lonstein and Winter [30] found a relationship between the final outcome of brace treatment and curve factors and factors that predict future skeletal growth. In biomechanical theories describing the patho-physiological mechanism of scoliosis, spinal growth is thought to be the main driving force responsible for curve progression [35, 42, 44, 47, 48].

Throughout history, external devices have been used to correct deformities and immobilize the spine. Brace designs have changed periodically over the years, but most modifications have solely focused on improved efficacy and failed to acknowledge the importance, especially to teenagers, of physical appearance. This age group resists acting or looking different from their peers, which obviously occurs when a visible brace is worn. Modern materials, lower profiles, and reduced wearing times have been tried in attempts to reduce resistance to and the emotional difficulties encountered with wearing braces.

The aim in the design of a new orthosis was to create a system which is at least as effective as the TLSO's, with improved comfort for the wearer. The newly introduced brace should reverse the transverse force pattern by externally applied and constantly present orthotic forces without limiting the normal body motions of the patient and an increased comfort level for the patient.

\section{Construction and working principle of the TriaC} orthosis

The name TriaC is based on the three C's of Comfort, Control, and Cosmetics. Currently, the orthosis is produced by Somas International, St Anthonis, the Netherlands. For this orthosis, the choice has been made to apply continuous correction forces on the chest with the aim to prevent curve progression during the growth period. A basic requirement for such a brace is that the brace force must be able to follow the main body motions of the patient. To achieve this goal required the use of a flexible coupling, connecting the thoracic and lumbar parts of the orthosis. The thoracic part and lumbar sections each generate their own corrective force on the body of the patient. Both parts are connected to each other by a flexible coupling that is placed on the opposite side of the thoracic force pad (Fig. 1). This coupling enables the patient to bend forward, backward and sideways while the correction forces are maintained (Fig. 2). The thoracic force pad is located just below the shoulder blade and applies a corrective force in a lateralanterior direction. The lumbar force pad is placed between the pelvis and the lower ribs and acts on the lumbar muscles in a lateral direction. A third counter force on the hip region ensures that the orthosis is in equilibrium.

Placing a flexible coupling between the thoracic and the lumbar part of the orthosis determines the unique force pattern on the patient both with respect to the location of the forces as with respect to the relation between the forces (Fig. 2). Modelling and clinical use have shown that the TriaC is suitable for all curve types, with the exception of curves with an apex at the 12th thoracic and the 1st lumbar vertebra. For these curve type the Lumbar force pad must be placed at the location of the flexible coupling which is not possible in the current construction of the orthosis. The construction as described, makes the orthosis force driven. When the patient moves her or his body in any direction (e.g. flexion, extension, rotation or lateral bending), the force pattern exerted by the orthosis moves along with the patient and thus is maintained constant. For this reason we defined the orthosis as "dynamic" because the orthosis moves dynamically along with the body motions of the patient.

In contrast, conventional orthoses are displacement driven. A rigid shell shaped in the desired corrected position of the patient applies a corrective force only when the thorax of the patient presses against the pads of the brace.

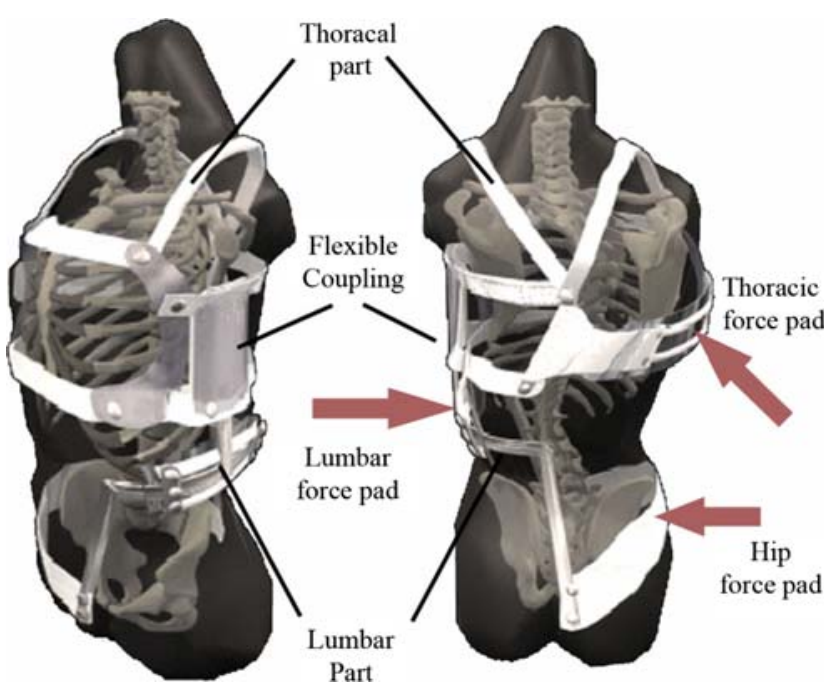

Fig. 1 The TriaC orthosis, with a thoracic part, a lumbar part and a flexible coupling 


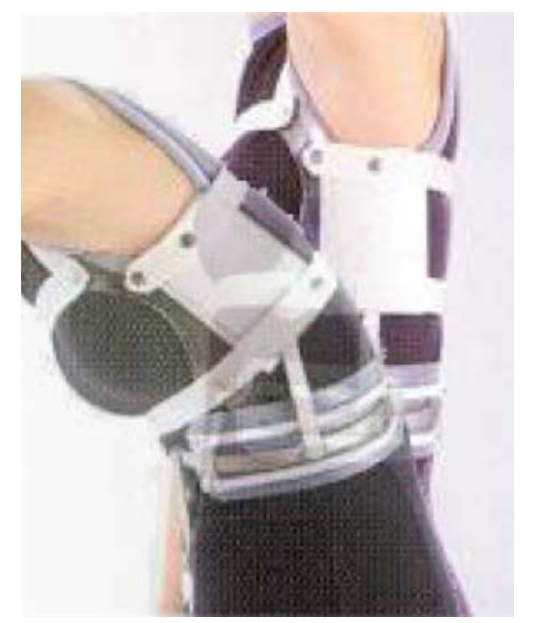

Fig. 2 The flexible coupling

When the body of the patient moves away from these pads the rigid brace is not able to follow the body motion of the patient and therefore the brace no longer generates forces on the patient. Therefore, we defined these orthosis as "static".

\section{Materials and methods}

From 1997 to 2005 all consecutive patients who met the inclusion criteria were treated nonoperatively with the TriaC orthosis and studied prospectively. The indication for treatment was a progressive curve with a Cobb angle between 25 and $40^{\circ}$. All curves, except curves already at $>30^{\circ}$, had to show documented progression of at least $5^{\circ}$. Patients had to be skeletally immature, defined as a Risser sign 0 or 1 for both boys and girls; in addition, girls are premenarche or less than 1-year post-menarche. All the curves had to be flexible as demonstrated by at least a $40 \%$ correction on the bending radiograph at the first visit. Patients with the apex of the curve at T12 and L1 were excluded, as were patients with a systemic disease that could possibly influence the outcome of the study (Table 1).

All patients who met the in- and exclusion criteria were treated with the TriaC orthosis. An informed consent form was obtained from all parents of the subjects in regard of the treatment with a new orthosis. According to the medical ethics committee no informed consent was necessary in case of the follow-up schedule because there is no difference with the patients treated with a Boston brace in our clinic.

If during the treatment period the orthosis failed to stop progression and the curve increased significantly, the patient was placed into a Boston Brace. This procedure was chosen to get a first indication whether a Boston brace
Table 1 The in and exclusion criteria for the treatment with the TriaC orthosis

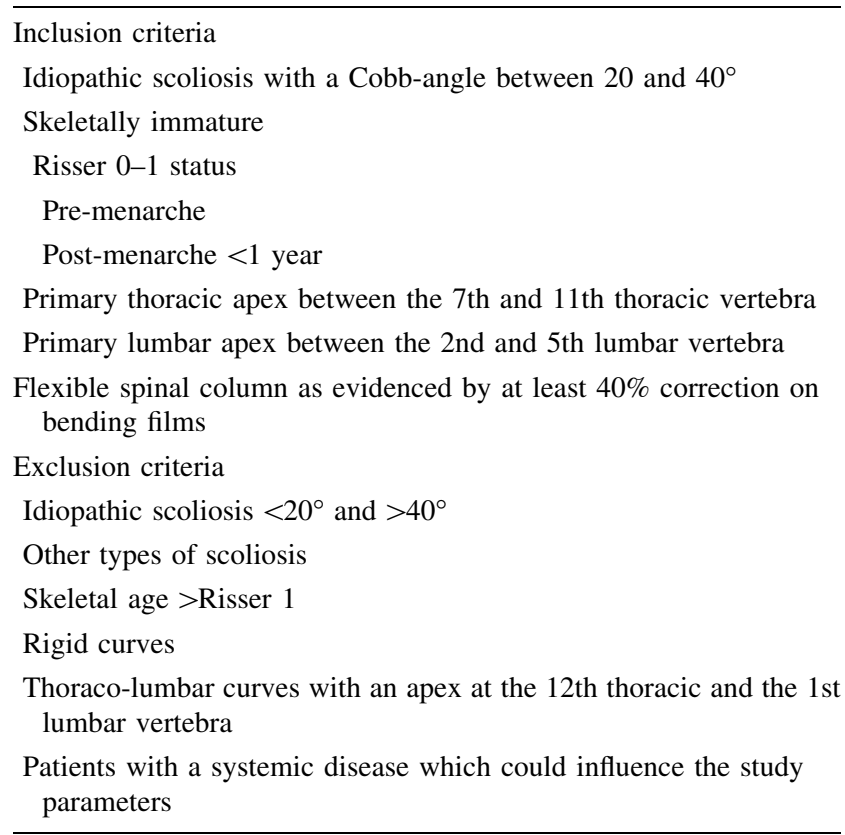

would be able to prevent curve progression in patients where the TriaC failed.

Failure was defined as either a Cobb angle of $>45^{\circ}$, i.e. the generally accepted indication for surgery, or if the curve progressed $5^{\circ}$ or more compared with the measurement at study entry [16, 24-31]. During the nonoperative treatment patients were checked by an orthotist at the outpatient clinic every 4 months, where the subjective compliance was documented. At every visit a PA and lateral radiographs were made. The patients were allowed to wean of the braces at skeletally maturity, i.e. when the radiographs showed Risser sign 4 or, for girls, 2 years postmenarche and patients did not show any further growth at length measurements. At every visit standing and sitting height of the patient was recorded as an additional measurement, and these had to show no further increase. Because the TriaC is designed as a dynamic orthosis, it possibly preserves the muscle status of the patient. As a result the weaning process could be faster than with rigid Boston type orthoses. In this study, weaning was generally complete within 4 weeks, whereas for rigid braces this process may take up to one year. After terminating the treatment patients were seen once a year with an AP and lateral X-ray.

\section{Radiographic analysis}

All radiographs were obtained in a standing position. The posterior-anterior projection was used to minimize the 
radiation dose to the breast. All radiographs were made, using a standardized protocol. Anatomical vertebral landmarks are identified and manually marked on a $21 \mathrm{in.} \mathrm{high}$ resolution monitor using a pointer [46]. Following the placement of the markers the Cobb angle is calculated, using automatic detection of the apex and end vertebrae [8].

In this article, only the Cobb-angle data are used for comparison with literature data on the natural history.

\section{Results}

Out of a total of 212 patients that were treated at the University Hospital of Groningen during the study period, 63 , or $30 \%$, met all the in- and exclusion criteria and form the study group. There were seven patients $(11 \%)$ with a single curve and 56 (89\%) with a double curve. The group included six boys and 57 girls. The mean age of the patients at the start of treatment was $11.3 \pm 3.1$ years. The treatment was complete when the patients had reached Risser 4 with a mean age of $15.6 \pm 1.1$ years. The mean Cobb angle of the primary curves before the brace treatment was started was $30.2^{\circ} \pm 7.5^{\circ}$. The secondary curves had a mean Cobb angle of $22.3^{\circ} \pm 6.4^{\circ}$.

The distribution of the location of the apex vertebra of the primary curves is shown in Fig. 3.

The patient group was divided into a success group and a failure group whereby failure was defined as a Cobb angle of $>45^{\circ}$ or curve progression of more than $5^{\circ}$ compared to baseline $[2,7,9,18,21,33,34,36,40]$.

The X-rays showed a mean initial correction of $22 \pm 26 \%$ for the primary curves, and a mean correction of and $28 \pm 35 \%$ for the secondary curves. The large variation was primarily caused by the big difference between the initial corrections seen in patients that were considered a success in comparison to patients that were considered a

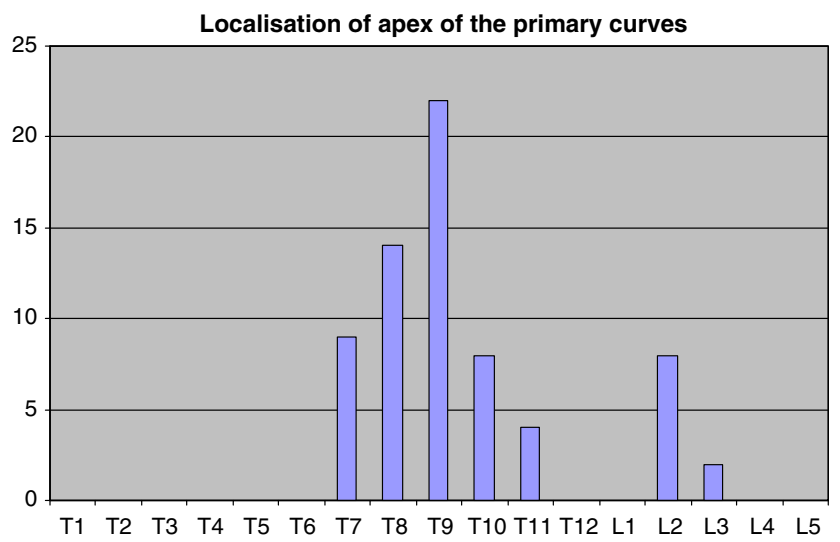

Fig. 3 The distribution of the apex level of the primary curves in the study group failure. In the success group the initial correction of the primary curve was $34 \pm 17 \%$ whereas the initial correction in the failure group was $-16 \pm 17 \%$. For the entire study group the success rate is $76 \%$ (48 successes) for a failure rate of $24 \%$ ( 15 failures). In the single curve group the mean Cobb angle at start of the treatment was $35^{\circ} \pm 11$. The mean initial correction achieved in this group was $23 \pm 9 \%$. There were no failures in this group. Although this difference is remarkable compared to the double curved group the single curve group is too small to draw conclusions.

In Table 2 an overview of the success rate is shown, divided per initial Risser sub group (Risser 0 and Risser 1) and divided by the initial Cobb angle at start of the treatment.

The actual number of failures in our patient population was 15 out of 63 patients, or $24 \%$. All patients in whom the brace failed went on to surgery, including the nine patients who were also treated with a Boston style brace after the TriaC brace had failed. A more detailed view of the correction of the primary Cobb angle is presented in Fig. 4. In this figure the mean correction percentage of the Cobb angle is shown as a function of the percentage of the total treatment brace period. The treatment period is considered to be at $0 \%$ at the start of the treatment and at $100 \%$ at the time the brace is discontinued.

It is shown that it was not possible to completely maintain the initial correction for the whole treatment period. At the end of treatment, the mean correction of the Cobb angle in the success patients had decreased to $19 \pm 13 \%$. This decrease in correction does not appear to follow a linear pattern. When a relation between the time that the patient wears the brace, and the loss of correction in the brace is assumed the correlation is only (very) weak $(\rho=-0.33)$. The mean correction at the first visit after discontinuation of the brace the treatment was $20 \pm 15 \%$. All patients in whom the brace treatment was a success were followed with a mean follow-up of 1.6 years and longest follow-up of 6 years post-bracing. Figure 5 shows the mean correction at different follow-up periods postbracing.

Because the orthosis does not affect the lateral curvature no significant changes were expected. The analysis of the sagittal curve is displayed in Fig. 6.

Table 2 Number of successes/total patients in subgroups

\begin{tabular}{lll}
\hline Cobb angle & \multicolumn{2}{l}{ Success rate } \\
\cline { 2 - 3 } & Risser 0 & Risser 1 \\
\hline $20-29^{\circ}$ & $76 \%$ (16 of 21 patients) & \\
$30-39^{\circ}$ & $74 \%$ (26 of 35 patients) & $86 \%$ (6 of 7 patients) \\
Total & $75 \%$ (42 of 65 patients) & $86 \%$ (6 of 7 patients) \\
\hline
\end{tabular}




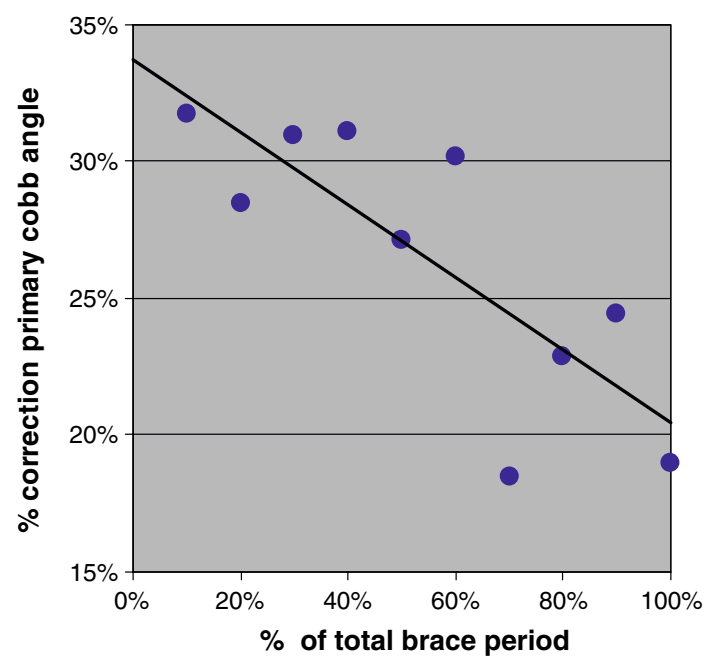

Fig. 4 Percent correction of the Cobb angle over the entire TriaC treatment period in the success group

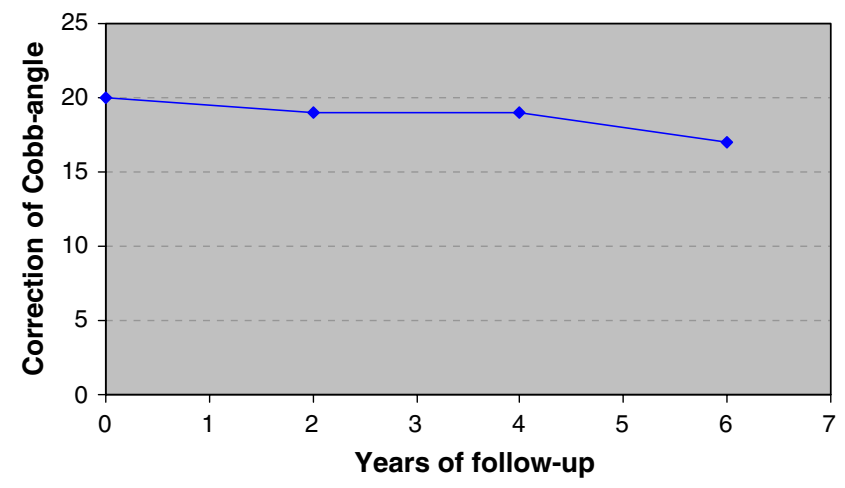

Fig. 5 The mean correction during follow-up after termination of successful brace treatment

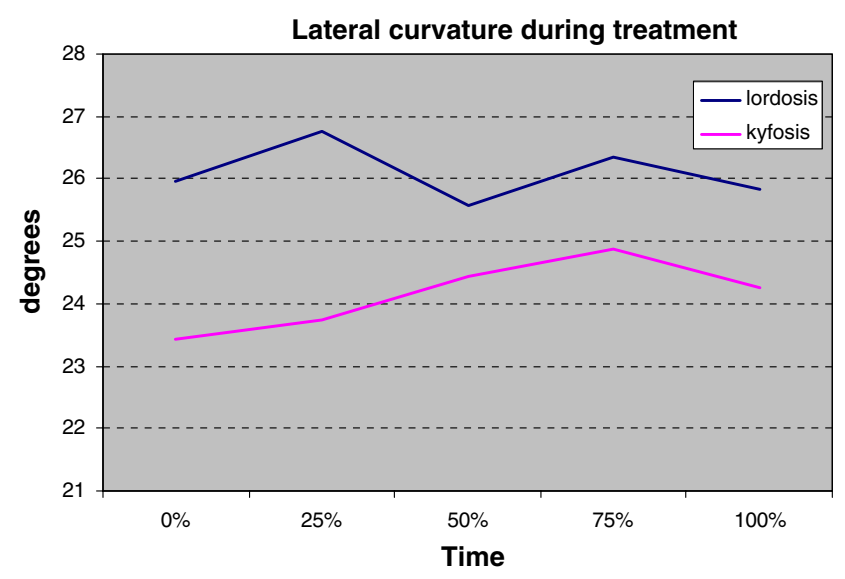

Fig. 6 Lateral curve during treatment

\section{Discussion}

Braces are the oldest recorded method of treatment for spinal injury and deformity. The primary goal in treating patients with scoliosis deformities is to stabilize the curves to prevent further progression of the deformity. Closely related to this is the goal of achieving correction of the spinal deformity, although this is not part of the therapeutic regimen for every patient. Brace treatments do not generally correct the scoliosis, but prevent further progression, i.e. bracing has a "holding effect" [49]. In most published studies, the brace treatment has been considered a failure if the patient needed operative stabilisation or if the curve progressed $5^{\circ}$ or more after the initiation of treatment $[2,7$, $9,18,21,30,33,34,40]$. However, these should not be the only criteria to determine whether brace treatment is successful. The treatment should also improve the patient's outcome when compared with the expected natural history.

The aim of this study was to investigate whether the TriaC-brace affects the natural history of idiopathic scoliosis. Bunnell [3], studied the natural history of idiopathic scoliosis in a group of patients with similar characteristics to our study group with respect to curve magnitude and age at the first visit. He showed that for the group as a whole $68 \%$ show a curve progression of $5^{\circ}$ or more and only $34 \%$ of the patients showed a curve progression of $10^{\circ}$ or more. The latter progression rate is in the same range as the failure rate reported in most of the retrospective studies, regarding overall brace effectiveness. However, the progression rates are not equal for every sub group of scoliotic patients. Lonstein and Carlson [29] reported that patients with Risser 0 or 1 maturity and curves larger than $20^{\circ}$ are three times more likely to experience curve progression than those with curves smaller than $20^{\circ}$. For those with curves exceeding $20^{\circ}$, patients with Risser 0 or 1 maturity also are three times more likely to experience curve progression than are patients with Risser 2-5 maturity. They classified Risser 0-1 patients with curves between 20 and $29^{\circ}$ as high risk (40-70\%) and Risser 0-1 patients with curves between 29 and 39 as very high risk (70-90\%). Bunnell [3] also found Risser sign and curve size to be good prognostic factors for curve progression in untreated idiopathic scoliosis. In his series patients with a Risser 0 at the time of diagnosis had a $68 \%$ risk of progression $10^{\circ}$ or more. This risk was decreased to $52 \%$ in those who had a Risser sign of 1 or 2 and was further decreased to $18 \%$ for those with Risser 3 or 4 . Nachemson et al. [36] reported that $66 \%$ of skeletally immature female patients with untreated idiopathic curves between $25^{\circ}$ and $35^{\circ}$ will experience curve progression greater than 5 .

All patients in our study started at Risser 0-1 maturity and had a curve above $30^{\circ}$ or, if they had an initial curve below $30^{\circ}$ had to show documented progression of $\geq 5^{\circ}$ 
before enrolment in this study. Wever et al. [47] showed that there is a strong correlation between the curve progression and spinal growth of the patients. Usually the spinal growth is highest around the beginning of the menarche and therefore we only included female patients when they were before or less than 1 year after menarche. By using these inclusion criteria we aimed to include only patients at the highest risk for curve progression.

In order to evaluate whether the TriaC brace alters the natural history of the high-risk patients, we estimated the natural progression rate of our patient group based on the literature as discussed. Our estimates are based on the calculation of a progression factor for all patients in the cohort as suggested by Lonstein and Carlson [29]. According to their algorithm, all our patients individually would have a change of $70-100 \%$ of progression of their curves. Based on these data we chose the low end of this range and assumed that in our patient group $70 \%$ of the curves would progress without treatment. The actual number of failures in our patient population was 15 out of 63 patients, or $24 \%$, which is a significantly different from the expected number of $44(P<0.001)$.

The validity of the sample size was evaluated by calculating the $95 \%$ confidence interval for the failure rate, which was between 11 and $36 \%$ (7-23 patients). Thus, the difference in outcomes remains statistically significant. These data show that the TriaC brace significantly altered the predicted natural history of curve progression. The comparison of the results of this study and other brace studies reported in the literature is difficult because of differences in sample size, skeletal maturity and curve magnitudes at the initiation of bracing. Furthermore, some reports excluded those patients who were not compliant with their bracing regimen. These large variations render the value of a statistical comparison of the results doubtful [51]. By applying the inclusion criteria used in this study to the literature data, a more uniform group has been created, making the statistical comparison more reliable. Nachemson et al. [36] prospectively compared 111 adolescent idiopathic scoliotic female patients with curves between $25^{\circ}$ and $35^{\circ}$, who were treated with an underarm plastic brace, to 129 patients who were not treated. All patients were followed for 4 years. The patient's thoracic or thoracolumbar curves were during this period evaluated for curve progression of more than $5^{\circ}$. Of the brace treated patients, 23 patients where lost to follow-up. Using survivorship analysis, a successful outcome was estimated in $74 \%$ of patients treated with a brace, compared to $34 \%$ of those who had no treatment; this difference was significant $(P<0.0001)$. In a retrospective study Lonstein and Winter [30] evaluated the result of brace treatment in a group of 1,020 patients over 35 years. From these patients a sub group of 177 had a curve between 20 and $29^{\circ}$ and a Risser sign of $0-1$. Using the natural history data of a similar group of non treated patients from their previous report [29] they where able to show in this sub-group a significant difference ( $P=0.0001$, chi-square test) of the failure rate between the group of treated patients and not treated patients. The not treated patients had a predicted failure rate of $68 \%$ and the failure rate of the braced patients in the sub group was $40 \%$. We used the same report to predict the failure rate in our cohort. Although our results are promising, it is known that $5 \%$ of patients with a curve of less than $30^{\circ}$ still progress to surgery during mature life [34]. We will follow our patients in whom the TriaC treatment effectively stopped curve progression before the surgical cut-off of $45^{\circ}$ during mature life.

Besides affecting the lateral curve, most traditional braces reduce lordosis and kyphosis by tilting the pelvis. The purpose of pelvic tilt is to move the lumbar spine closer to the correction pads within the brace. According to Lindh et al. [28] reducing the lumbar lordosis may automatically lead to a reduction of the scoliosis as a result of a coupling mechanism between sagittal and lateral motions of the vertebra [38]. However, reduction of lumbar lordosis will also reduce thoracic kyphosis. The reduction of thoracic kyphosis is an unwanted effect, as reduced thoracic kyphosis already is an integral component of the scoliotic deformity [50]. Schaal et al. therefore, emphasise the need for a system that diminishes the effect of pelvic tilt on the thoracic kyphosis [43]. In the Triac brace the choice has been made to continuously apply corrective forces with the aim of reversing the deforming forces, without affecting the lordosis of the spine [37]. Growth is a continuous process and therefore, the correction forces should be applied continuously as well, even during the normal body motions of the patient.

In order to meet this requirement a flexible coupling, connecting the thoracic and lumbar parts of the brace is incorporated in the device. Most importantly, the forces exerted by the brace must be applied in such a way that they are maintained during all body motions of the patient. The new brace incorporates three separate functional elements: frame, springs and pelottes. The springs generate the orthotic forces, which are distributed by the frame and transmitted to the skin by the pelottes. The flexible coupling connects the thoracic and lumbar frame parts resulting in the application of constant forces that cannot be modified by the patients themselves (Fig. 7). The strength of the externally applied forces was chosen on the basis of literature data [10, 20, 27] Fig. 8.

The TriaC-brace provides a good primary correction of idiopathic scoliosis. There was a mean initial correction within the brace of $22 \pm 26 \%$ for the primary curves and $28 \pm 35 \%$ for the secondary curves, which is slightly less than reported in the literature $[2,30,33,34]$. In the 
Fig. 7 TriaC-brace
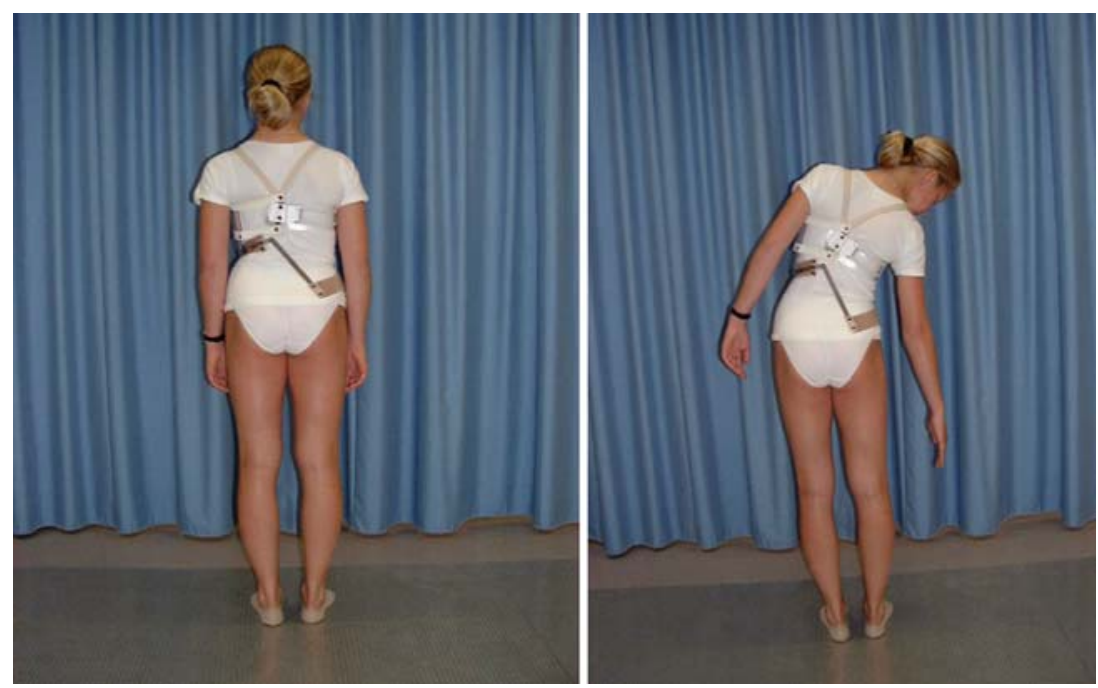

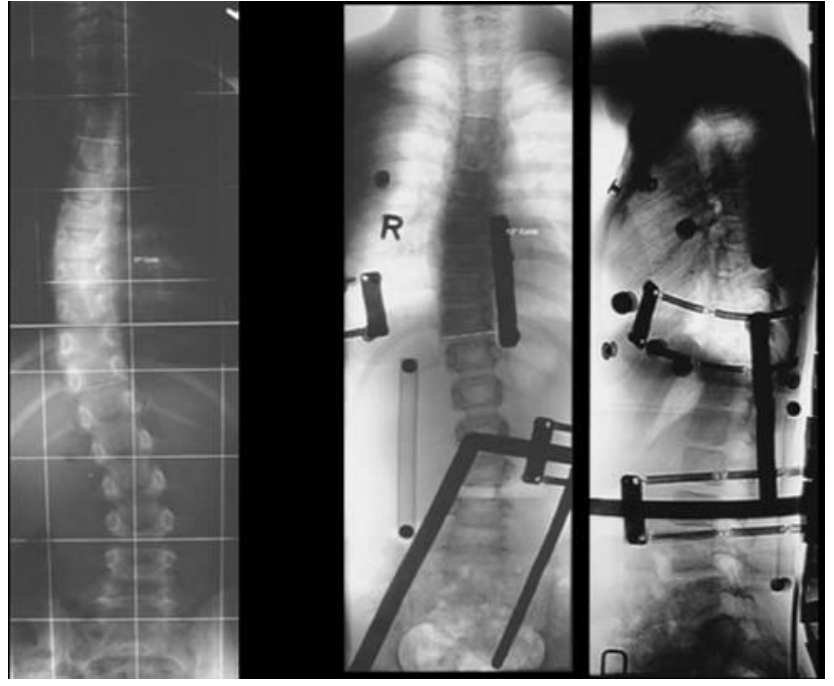

Fig. 8 Pre-brace Cobb-angle: $30^{\circ}$ and $19^{\circ}$ apical axial rotation. In the brace Cobb-angle: $17^{\circ}$ and $9^{\circ}$ apical axial rotation, sagittal curvature unchanged

beginning of the study, we were not certain how patients would respond to the application of constant forces. Therefore, we started very carefully using relatively low forces. As we found that patients tolerated these forces well we subsequently increased the amount of force which resulted in improved initial corrections. According to the literature, achieved corrections gradually are lost following the termination of the brace treatment [7, 23, 30, 34, 49].

However, being efficacious is not all that is required of a brace; it should also be as comfortable as possible and cosmetically acceptable so that teenagers who require this treatment will actually use it. Non-acceptance of a brace by patients is a real and serious problem. Houghton et al. [24] placed a hidden transducer in their braces and found that actual compliance was considerably less than was reported by the patient; and only $20 \%$ of patients wore the brace as prescribed. Modern materials, lower profiles and reduced wearing times have all been tried to improve compliance and reduce the emotional difficulties experienced with brace wear. According to some authors, there is little difference in effect between part-time (12-16 h) and full-time $(23 \mathrm{~h})$ wearing of a brace [13, 21]. Kahanowitz [25] reported such findings but only if the pre-brace Cobb-angle were less than $35^{\circ}$; if larger than this more than $50 \%$ of curves would progress to such an extent that surgery became necessary.

The current study demonstrates that the TriaC brace reduces the scoliosis, and the achieved correction is maintained during brace treatment. It prevents further progression of the Cobb angle in idiopathic scoliosis. The new brace does not differ from the conventional braces as far as maintaining the deformity in the coronal plane is concerned. This is expected as the force system of the TriaC brace in the frontal plane is in accordance with the force system in the conventional braces.

The new brace offers more comfort to the patient and a better cosmetic appearance. With TriaC brace there are no restrictions regarding daily or sporting activities, and it can be worn with all types of clothing.

\section{Summary}

Compared with published studies on the natural history of idiopathic scoliosis, use of the Triac brace appeared to significantly improve the course of curves between 20 and $40^{\circ}$ in skeletally immature individuals. Control or net correction of idiopathic scoliotic curves was achieved in $76 \%$ of patients.

The new dynamic brace offers more comfort to the patient and a better cosmetic appearance. 
Acknowledgments The authors would like to thank Persenaire JM, MD and Webb PJ, FRCS for their critical remarks and advice.

\section{References}

1. Andriacchi TP, Schultz AB, Belytschko TB, DeWald RL (1976) Milwaukee brace correction of idiopathic scoliosis. J Bone Joint Surg Am 58:806-815

2. Bassett GS, Bunnell WP, MacEwen GD (1986) Treatment of idiopathic scoliosis with the Wilmington brace. Results in patients with a twenty to thirty-nine degree curve. J Bone Joint Surg Am 68:602-625

3. Bunnell WP (1986) The natural history of idiopathic scoliosis before skeletal maturity. Spine 11:773-776

4. Bunnell WP, MacEwen GD, Jayakumar S (1980) The use of plastic jackets in the nonoperative treatment of idiopathic scoliosis: preliminary report. J Bone Joint Surg Am 62:31-38

5. Burwell RG (1971) The relationship between scoliosis, growth In: Zorab PA (eds) Scoliosis and Growth. Edinburgh, Churchill Livingstone, pp 131-150

6. Calvo IJ (1955) Observations on the growth of the female adolescent spine and its relation to scoliosis. Clin Orthop 10:40-47

7. Carr WA, Moe JH, Winter RB, Lonstein JE (1980) Treatment of idiopathic scoliosis in the Milwaukee brace. Long-term results. J Bone Joint Surg Am 62:599-612

8. Cheung J, Wever DJ, Veldhuizen AG, Klein JP, Verdonck B, Nijlunsung R, Cool JC, Horn JR (2002) The reliability of quantitative analysis of digital images of the scoliosis. Eur Spine $\mathrm{J}$ $11: 535-542$

9. Cochran T, Nachemson A (1985) Long-term anatomic and functional changes in patients with adolescent idiopathic scoliosis treated with the Milwaukee brace. Spine 10:127-133

10. Cochran GVB, Waugh TR (1969) The external forces in correction of idiopathic scoliosis. In: Proceedings of the scoliosis. J Bone Joint Surg (AM) 51:201

11. Dickson RA, Deacon P (1987) Spinal growth. J Bone Joint Surg Br 69:690-692

12. Dickson RA (1985) Conservative treatment for idiopathic scoliosis. J Bone Joint Surg Br 67:176-181

13. DiRaimondo CV, Green NE (1988) Brace-wear compliance in patients with adolescent idiopathic scoliosis. J Pediatr Orthop 8:143-146

14. Duval-Beaupere G (1971) Pathogenic relationship between scoliosis and growth. In: Zorab PA (eds) Scoliosis and growth. Edinburgh, Churchill Livingstone, pp 58-61

15. Duval-Beaupere G, Lamireau T (1985) Scoliosis at less than 30 degrees. Properties of the evolutivity (risk of progression). Spine 10:421-424

16. Edgar MA (1985) To brace or not to brace? J Bone Joint Surg Br 67:173-174

17. Emans JB, Kaelin A, Bancel P, Hall JE, Miller Me (1986) The Boston bracing system for idiopathic scoliosis: a follow-up results in 295 patients. Spine 11:792-801

18. Fisher DA, Rapp GF, Emkes M (1987) Idiopathic scoliosis: transcutaneous muscle stimulation versus the Milwaukee brace. Spine 12:792-801

19. Focarile FA, Bonaldi A, Giarolo MA et al (1991) Effectiveness of nonsurgical treatment for idiopathic scoliosis. Overview of available evidence. Spine 16:395-401

20. Galante J, Schultz AB, deWald RL, Ray RD (1970) Forces acting in the Milwaukee brace in patients under treatment for idiopathic scoliosis. J Bone Joint Surg AM 52:498-506

21. Green NE (1986) Part-time bracing of adolescent idiopathic scoliosis. J Bone Joint Surg AM 68:738-42
22. Hanks GA, Zimmer B, Nogi J (1988) TLSO treatment of idiopathic scoliosis. An analysis of the Wilmington Jacket. Spine 13:626-629

23. Hassan I, Bjerkreim I (1983) Progression in idiopathic scoliosis after conservative treatment. Acta Orthop Scand 54:88-90

24. Houghton GR, McInerney A, Tew T (1987) Monitoring true brace compliance. Orthop Trans 11:105

25. Kahanowitz N, Snow B, Pinter I (1984) The comparative results of psychologic testing inscoliosis patients treated with electric stimulation or bracing. Spine 9:442-444

26. Katz DE, Richards BS, Browne RH, Herring JA (1997) A comparison between the Boston brace and the Charleston Bending brace in adolescent Idiopathic scoliosis Spine 22:1302-1312

27. Lindahl O, Raeder E (1962) Mechanical analysis of the forces involved in idiopathic scoliosis. Acta Orthop Scand 32:27-38

28. Lindh M (1980) The effect of sagittal curve changes on brace correction of idiopathicscoliosis. Spine 5:26-36

29. Lonstein JE, Carlson JM (1984) The prediction of curve progression in untreated idiopathic scoliosis during growth. J Bone Joint Surg Am 66:1061-1071

30. Lonstein JE, Winter RB (1994) The Milwaukee brace for the treatment of adolescent idiopathic scoliosis. A review of one thousand and twenty patients. J Bone Joint Surg Am 76:12071221

31. McCollough NC, Schultz M, Javeck N, Latta L (1981) Miami TLSO in the management of scoliosis: preliminary results in 100 cases. J Ped Orthop 1:141-152

32. Miller JAA, Nachemson Al, Schultz AB (1984) Effectiveness of braces in mild idiopathic scoliosis. Spine 9:632-635

33. Moe JH, Kettelson DN (1970) Idiopathic scoliosis. Analysis of curve patterns and the preliminary results of Milwaukee-brace treatment in one hundred and sixty-nine patients. J Bone Joint Surg Am 52:1509-1533

34. Montgomery F, Willner S (1989) Prognosis of brace-treated scoliosis. Comparison of the Boston and Milwaukee methods in 244 girls. Acta Orthop Scand 60:383-385

35. Murray DW, Bulstrode CJ (1996) The development of idiopathic scoliosis. Eur Spine J 5:251-257

36. Nachemson AL, Peterson LE, members of the Brace Study Group of the Scoliosis Research Society (1995) Effectiveness of treatment with a brace in girls who have adolescent idiopathic scoliosis. J Bone Joint Surg Am 77:815-22

37. Nijenbanning G (1998) Scoliosis redress design of a force controlled Orthosis. Thesis, Universiteit Twente, Enschede, FEBO, The Netherlands. ISBN 90-36511925

38. Ogilvie J (1994) Spinal orthotics: an overview. In: Weinstein SL (ed) The pedriatric spine principle and practice, Chap 84, Raven Press, New York, pp 1787-1793

39. Peterson LE, Nachemson AL (1995) Prediction of progression of the curve in girls who have adolescent idiopathic scoliosis of moderate severity. Logistic regression analysis based on data from The Brace Study of the Scoliosis Research Soc. J Bone Joint Surg Am 77:823-827

40. Price CT, Scott DS, Reed FE, Riddick MF (1990) Nighttime bracing for adolescent idiopathic scoliosis with the Charleston bending brace. Preliminary report. Spine 15:1294-1299

41. Risser JC, Ferguson AB (1936) Scoliosis: its prognosis. J Bone Joint Surg Am 18:667-670

42. Roaf R (1960) Vertebral growth and its mechanical control. J Bone Joint Surg Br 42:40-59

43. Schaal A, Chêneau J (1990) Aktueller stand des ständig verbesserten Chêneau Korsettes Orthopädie Technik 4:213-216

44. Stokes IAF, Spence H, Aronsson DD, Kilmer N (1996) Mechanical modulation of vertebral body growth. Implications for scoliosis progression. Spine 21:1162-1167 
45. Veldhuizen AG, Cheung J, Bulthuis GJ, Nijenbanning G (2002) A new orthotic device in the non-operative treatment of idiopathic scoliosis. Med Eng Phys 24:209-218

46. Verdonck B, Nijlunsing R, Gerritsen FA, Cheung J, Wever DJ, Veldhuizen AG, Devillers S, Makram-Ebeld S (1998) Computer assisted quantitative analysis of deformations of the human spine. Proceedings MICCAI conference. Lect Notes Comput Sci 1496:822-831

47. Wever DJ, Tonseth KA, Veldhuizen AG, Cool JC, v.Horn JR (2000) Curve progression and spinal growth in brace treated idiopathic scoliosis. Clin Orthop 377:169-179

48. Wever DJ, Veldhuizen AG, Klein JP, Webb PJ, Nijenbanning G, Cool JC, Horn JR (1999) A biomechanical analysis of the vertebral and rib deformities in structural scoliosis. Eur Spine $\mathbf{J}$ $8: 252-260$

49. Willner S (1988) Brace treatment of scoliosis. To treat or not to treat. In: Proceedings of the eighth Philip Zorab scoliosis symposium: prognosis in scoliosis, London. A.G. Bishop \& Sons Ltd, Orpington, Kent, pp 94-98

50. Willner S (1984) Effect of the Boston thoracic brace on the frontal and sagittal curves of the spine. Acta Orthop Scand 55:457-460

51. Winter RB, Lonstein JE, Drogt J, Noren CA (1986) THe effectiveness of bracing in the nonoperative treatment of idiopatic scoliosis. Spine 11:790-791 